\title{
Archivos \\ de Cardiología de Méxíco

\section{Por qué es importante lograr metas de hipertensión arterial sistémica... A propósito un caso clínico que inició como evento vascular cerebral isquémico}

\author{
Julio César Sauza-Sosa ${ }^{a, *}$, José Antonio Romero-Figueroa ${ }^{\mathrm{b}}$, \\ Lilia Mercedes Sierra-Galán ${ }^{a}$ y Sergio Mario Ferez-Santander ${ }^{c}$
}

\footnotetext{
a Servicio de Cardiología, Centro Médico American British Cowdray, Campus Santa Fe, México, D.F., México

b Servicio de Imagenología, Centro Médico American British Cowdray, Campus Santa Fe, México, D.F., México

c Servicio de Cardiología, Centro Médico American British Cowdray, Campus Observatorio, México, D.F., México
}

Recibido el 7 de abril de 2015; aceptado el 29 de septiembre de 2015

\author{
PALABRAS CLAVE \\ Hipertensión arterial; \\ Cardiopatía \\ hipertensiva; \\ Fibrilación auricular
}

\begin{abstract}
Resumen La hipertensión arterial sistémica (HAS) es una de las condiciones más frecuentes que se ve en atención primaria de las enfermedades cardiovasculares y que tiene como consecuencias; dependiendo del «órgano blanco» que afecte, producir la cardiopatía isquémica, la vasculopatía cerebral o la nefropatía crónica. Dentro de la patogénesis de la HAS se encuentran implicados varios mecanismos fisiopatológicos; de los cuales actualmente, por señalar los más importantes y frecuentes, juegan un papel el incremento en los niveles de adrenalina, el sistema renina-angiotensina-aldosterona y en fecha reciente, mucho se menciona la participación de la resistencia a la insulina y la hiperinsulinemia. Dichos procesos conllevan un desequilibrio entre el tono simpático y el parasimpático, aunado a la hipersensibilidad por el sodio desencadenan uno de los mecanismos fisiopatogénicos de la HAS.

Actualmente se define la HAS como el hallazgo de cifras de tensión arterial mayores a $140 / 90 \mathrm{~mm} \mathrm{Hg}$. Este es uno de los padecimientos que más afecta a la población mundial encontrando prevalencias en grupos etarios y de género de 45 al 55\% en varones entre los 45 y 70 años y del 45 al $65 \%$ en mujeres de ese mismo grupo etario.

En el 2013 se publicaron las guías clínicas más recientes para su tratamiento y las metas recomendadas, con lo que se ha logrado disminuir sus complicaciones y mortalidad; dentro de las que destacan enfermedades vasculares como la cardiopatía isquémica y la cerebral y renal. En el presente trabajo se comenta un caso clínico que ejemplifica las complicaciones secundarias en un diagnóstico tardío, el daño a «órgano blanco» por exposición a largo plazo y el inadecuado cumplimiento de las metas terapéuticas.

(c) 2015 Instituto Nacional de Cardiología Ignacio Chávez. Publicado por Masson Doyma México S.A. Todos los derechos reservados.
\end{abstract}

\footnotetext{
* Autor para correspondencia. Centro Médico ABC, Campus Santa Fe, Av. Carlos Graef Fernández 154-207, Col. Tlaxala, México, D.F., México, C.P. 05300.

Correo electrónico: sauzamd@hotmail.com (J.C. Sauza-Sosa).
} 


\section{KEYWORDS}

Arterial hypertension; Hypertensive heart disease

\section{Introducción}

La hipertensión arterial sistémica (HAS) es una de las condiciones más frecuentes que acude a la atención primaria de las enfermedades cardiocerebrovasculares y que tiene como consecuencias cardiopatía hipertensiva arterial sistémica (CHAS), cardiopatía isquémica, encefalopatía vascular cerebral (EVC), insuficiencia renal crónica y por todo ello la muerte $^{1}$. La meta del diagnóstico temprano y un tratamiento antihipertensivo adecuado, es disminuir la morbimortalidad por dichas enfermedades. En diciembre de 2013 tanto la European Society of Cardiology como el Eighth Joint National Commitee publicaron sus respectivas guías clínicas para el manejo de pacientes hipertensos las cuales se enfocaron en el diagnóstico, el tratamiento y las metas ${ }^{2}$.

Actualmente se define como un promedio de dos o más mediciones en por lo menos dos mediciones médicas por encima de $140 / 90 \mathrm{~mm} \mathrm{Hg}$, aunque también se sugiere realizar con la monitorización ambulatoria de las cifras de tensión arterial durante 24 o 48 horas y si las cifras de TA están por encima de $130 / 80 \mathrm{mmHg}$, promedio nocturno mayor de $120 / 70 \mathrm{mmHg}$ y promedio diurno mayor de 135/85 $\mathrm{mmHg}^{2,3}$.

La patogénesis de la HAS es poco clara. Una variedad de factores están implicados; dentro de los cuales se encuentran: raza, factores hereditarios, exceso de ingesta de sodio, de ingesta de alcohol, el sobrepeso corporal, el sedentarismo, la dislipidemia y la deficiencia de vitamina $\mathrm{D}^{4-6}$.

Dentro de las consecuencias que se encuentran secundarias al inadecuado manejo de la HAS, aparece la hipertrofia ventricular izquierda, la insuficiencia cardiaca, la fibrilación auricular (FA), EVC, la insuficiencia renal crónica y la aterosclerosis $^{7-10}$.
El tratamiento actual de la HAS se enfoca en alcanzar metas de cifras de TA de control con la terapéutica médica. El lograr dicha meta disminuye la morbimortalidad provocada de dicha enfermedad ${ }^{2,11}$.

\section{Caso clínico}

En el Servicio de Urgencias una paciente de 74 años ingresó por deterioro neurológico caracterizado por hiporreactividad secundaria y por infección de vías urinarias.

Previamente la paciente presentaba los antecedentes de diabetes mellitus (DMII), EVC de tipo isquémico con secuela de hemiparesia derecha en 2011, así como diagnóstico en ese mismo año de CHAS, de FA y descontrol de su hipertensión arterial sistémica. Cabe mencionar que en 2011 la paciente se conocía previamente solo con diagnóstico de DMII, por lo que en esta hospitalización se agregaron los diagnósticos previamente comentados.

A su ingreso la paciente presentó TA $170 / 95 \mathrm{~mm} \mathrm{Hg}$, frecuencia ventricular media $60 \mathrm{lpm}$, frecuencia respiratoria $18 \mathrm{rpm}$, temperatura $36^{\circ} \mathrm{C}$. Se encontró somnolienta, sin datos de focalización, fondo de ojo con arterias tortuosas, con venas distendidas y exudados retinianos grado III de Keith-Wagener-Baker. Ruidos cardiacos arrítmicos, con primer ruido de intensidad variable, segundo ruido con desdoblamiento fisiológico, no se auscultó tercer o cuarto ruido, con soplo de insuficiencia mitral y tricuspídea ॥/Iv. Abdomen asignológico. Extremidades íntegras con fuerza muscular conservada, reflejos osteotendinosos normales.

Los estudios de laboratorio con $\mathrm{Hb} 11.3 \mathrm{~g} / \mathrm{dL}$, Hto $34.2 \%$, leucocitos $16,500 / \mu \mathrm{l}$, segmentados $68 \%$, bandas $0 \%$, linfocitos $26 \%$, monocitos $5 \%$, plaquetas $299,000 / \mu l$, glucosa 


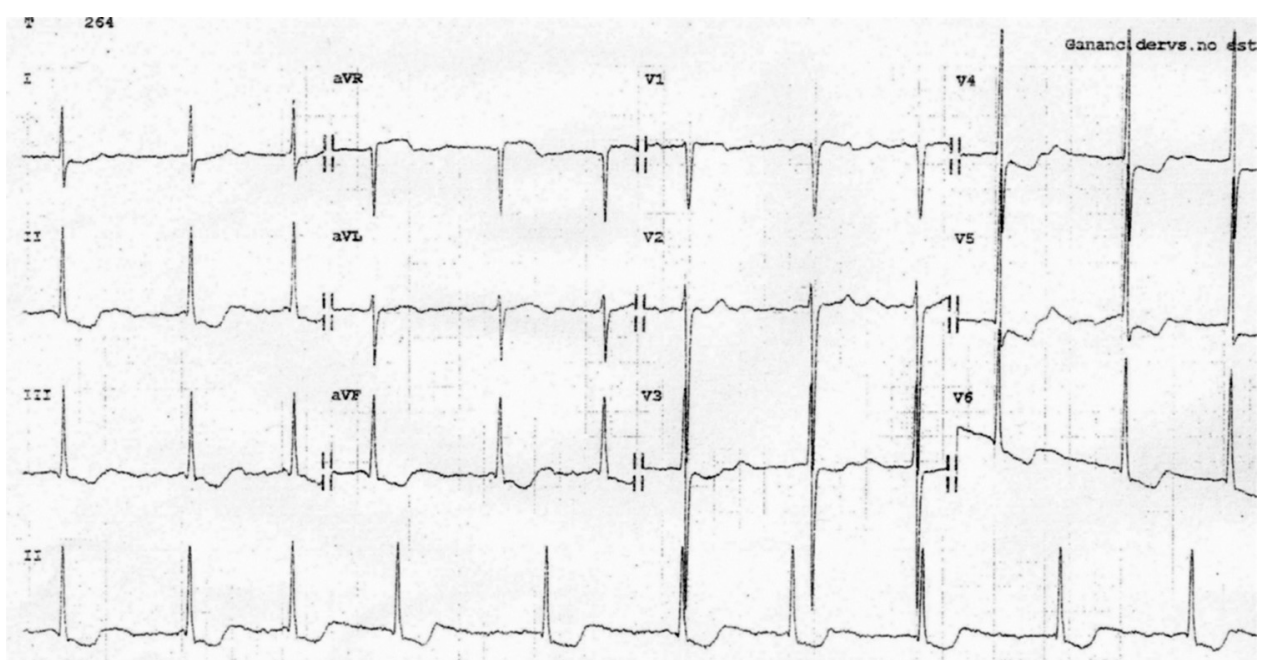

Figura 1 Fibrilación auricular con frecuencia ventricular media de 60-70 lpm, con datos de hipertrofia de ventrículo izquierdo (índice de Sokolow de $84 \mathrm{~mm}$ y de Cornell de $42 \mathrm{~mm}$ ) y de sobrecarga sistólica del mismo (desnivel negativo descendente del segmento S-T e inversión asimétrica de las ondas T en DII, DIII, AVF y de V3 a V6).

$202 \mathrm{mg} / \mathrm{dL}$, BUN $20 \mathrm{mg} / \mathrm{dL}$, creatinina $1.1 \mathrm{mg} / \mathrm{dL}$, colesterol $237 \mathrm{mg} / \mathrm{dL}$, AST $17 \mathrm{UI} / \mathrm{L}$, ALT $20 \mathrm{UI} / \mathrm{L}$, bilirrubina total $0.6 \mathrm{mg} / \mathrm{dL}$, bilirrubina directa $0.2 \mathrm{mg} / \mathrm{dL}$, bilirrubina indirecta $0.4 \mathrm{mg} / \mathrm{dL}$, proteínas totales $7.1 \mathrm{~g} / \mathrm{dL}$, albumina $4.2 \mathrm{~g} / \mathrm{dL}$, globulinas $2.9 \mathrm{~g} / \mathrm{dL}$, Na $135 \mathrm{mEq} / \mathrm{L}$, potasio 4.6 $\mathrm{mEq} / \mathrm{L}$, cloro $95 \mathrm{mEq} / \mathrm{L}$, ácido úrico $7.9 \mathrm{mg} / \mathrm{dL}, P C R$ $9.23 \mathrm{mg} / \mathrm{dL}$, albuminuria de $295 \mathrm{mg}$ en $24 \mathrm{~h}$.

El electrocardiograma mostró arritmia completa por fibrilación auricular con frecuencia cardiaca ventricular media de 70 lpm y datos de hipertrofia ventricular izquierda con sobrecarga sistólica (fig. 1).

Se realizó ecocardiograma transtorácico que mostró CHAS con HVI leve y dilatación de ambas aurículas (fig. 2).

La resonancia magnética de cerebro mostró zona de malacia por evento isquémico previo en región frontal izquierda así como cambios asociados a daño de la microvasculatura secundarios a HAS y DMII (fig. 3).

Es importante comentar que la paciente en 2011 comenzó con un EVC isquémico de origen cardioembólico, por una CHAS; así como datos crónicos de daño a sistema nervioso central secundarios a la HAS descompensada y sin lograr la optimización terapéutica para alcanzar las metas.

Presentamos una revisión de la literatura más actual sobre el diagnóstico, complicaciones, tratamiento y metas más recientemente establecidas.

\section{Definición y clasificación}

La definición de HAS surgió de varios estudios donde se correlacionó la interrelación entre las cifras elevadas de tensión arterial con las complicaciones cardiocerebro y renovasculares; de ahí es que se ha realizado el corte para el concepto universalmente aceptado para la definición de HAS y cifras de paciente normotenso ${ }^{12}$. Las últimas clasificaciones definen la HAS con cifras sistólicas de $\geq 140 \mathrm{~mm} \mathrm{Hg}$ y cifras diastólicas $\geq 90 \mathrm{~mm} \mathrm{Hg}$. La clasificación actual es (presiones sistólica/diastólica) óptimas de: $<120 /<80 \mathrm{~mm} \mathrm{Hg}$, normal: $120-129 / 80-84 \mathrm{~mm} \mathrm{Hg}$, normal alta: 130-139/85$89 \mathrm{~mm} \mathrm{Hg}$, HAS grado ।: 140-159/90-99 mm Hg, HAS grado ॥:
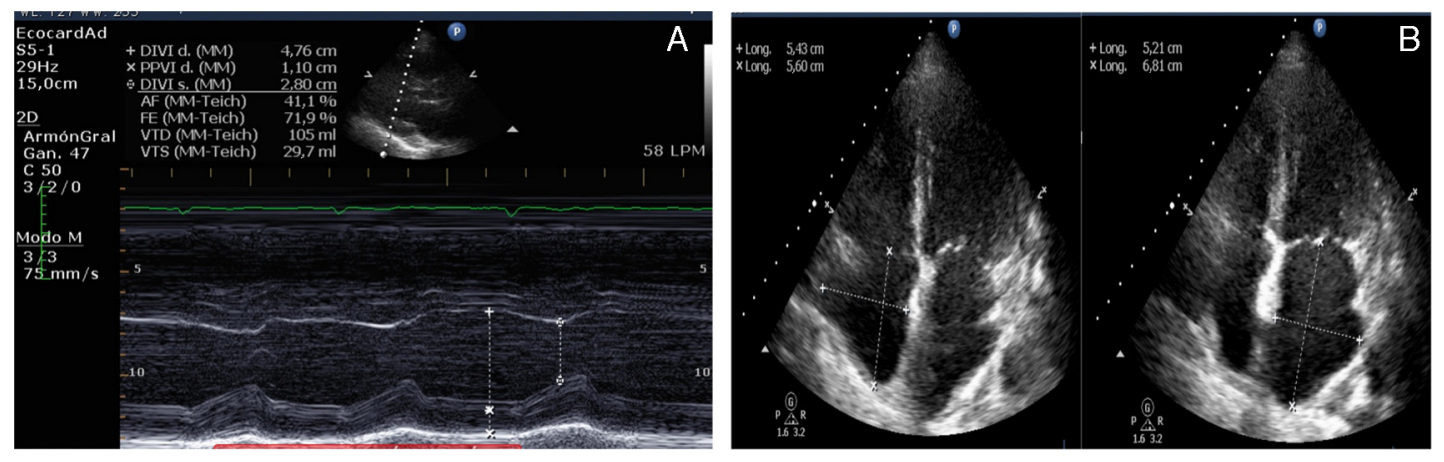

Figura 2 A) Eje paraesternal largo en modo $M$ que muestra hipertrofia leve de ventrículo izquierdo (pared posterior 11 mm) con diámetros intracavitarios dentro de límites normales. B) Eje apical de cuatro cámaras en modo bidimensional que muestra dilatación leve de ambas aurículas (aurícula derecha $5.4 \mathrm{~cm} \times 5.6 \mathrm{~cm}$ y aurícula izquierda $5.2 \mathrm{~cm} \times 6.8 \mathrm{~cm}$ ). 

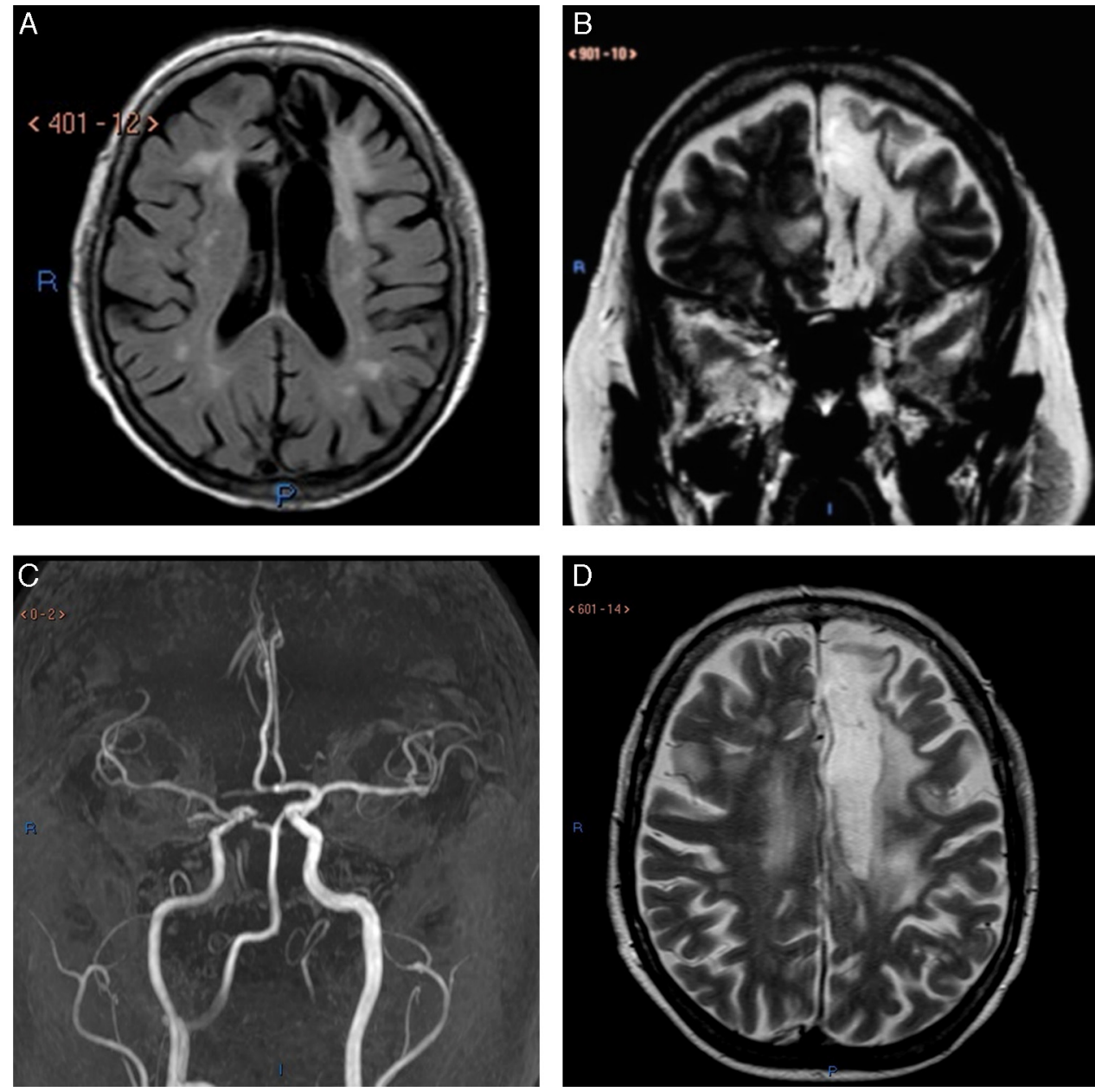

Figura 3 A) Corte axial al nivel de los ventrículos laterales que muestra área de malacia secuelar y gliosis frontomedial izquierda en el territorio de la arteria cerebral anterior. La sustancia blanca subcortical muestra hiperintensidades puntiformes compatibles con cambios micronagiopáticos. También hay dilatación del espacio subaracnoideo y de los ventrículos, asociado a atrofia. B) Plano coronal que muestra área de malacia secuelar que involucra ambas, sustancia blanca y gris cortical en el territorio de la arteria cerebral anterior, así como atrofia córtico subcortical. C) Muestra dominancia de la circulación carotídea e intracraneal izquierda que evidencia arteria cerebral media izquierda permeable, lo que sugiere que tuvo recanalización espontánea posterior al evento embólico cerebral antiguo. D) Plano axial al nivel de la corona radiada que muestra encefalomalacia frontal medial izquierda secuelar de evento vascular, así como edema y/o gliosis de la sustancia blanca bifrontal. También se observa dilatación de los espacios subaracnoideos asociado a decremento del volumen córtico subcortical.

160-179/100-109 mm Hg, HAS grado $11:>180 />110 \mathrm{~mm} \mathrm{Hg}$. Hipertensión sistólica aislada: $>140 /<90 \mathrm{~mm} \mathrm{Hg}^{11}$.

\section{Prevalencia}

La prevalencia varía en cada país; se presenta entre el 35$40 \%$ de la población general en grupos europeos ${ }^{11}$. En los Estados Unidos de América la HAS afecta a cerca de 78 millones de adultos. De ellos solo el $81.5 \%$ se encuentra diagnosticada, el $74.9 \%$ recibe tratamiento y solamente el $52.5 \%$ está controlado. Del total de pacientes no controlados solamente el $89.4 \%$ recibió una consulta médica. El costo estimado por esta enfermedad en Estados Unidos de América se calcula en 70 billones de dólares.

La HAS está presente en el $46 \%$ de los pacientes con diagnóstico de CHAS y en el $72 \%$ de los pacientes con EVC.

\section{Fisiopatología}

Actualmente se encuentran datos que sugieren que el incremento en las cifras de presión arterial que se encuentran en la fase de prehipertensión o HAS con cifras "limítrofes" altas son relacionadas a una vía que inicia con alteraciones genéticas (heredables); las cuales son responsables de una serie de cambios autonómicos y hemodinámicos ${ }^{13}$.

Dichos cambios consisten en un aumento en el tono simpático y disminución en el tono parasimpático; lo cual incrementa los niveles de norepinefrina plasmática. Debido a dicho incremento en catecolaminas se incrementa la frecuencia cardiaca, el dP/dT del ventrículo izquierdo (contractilidad); lo cual a su vez incrementa el índice cardiaco y es causante de una mala respuesta de las resistencias vasculares sistémicas; lo que causa un aumento en las cifras de presión arterial sistólica y diastólica ${ }^{13}$. 


\section{Diagnóstico}

Para realizar el diagnóstico de HAS lo primero es realizar una adecuada historia clínica, interrogando sobre antecedentes heredofamiliares y personales de esta enfermedad, dislipidémica, asociada a trastorno multivitamínico de los complejos B y D, homocisteinemia y DMII. Así como hábitos alimenticios, antecedente de tabaquismo y actividades físicas.

Además es importante interrogar sobre síntomas que indiquen daño a órgano blanco; como vértigo, cefalea, alteraciones visuales, alteraciones neurológicas, dolor torácico, disnea, síncope, palpitaciones, edema de extremidades inferiores, poliuria, hematuria, nicturia, microalbuminuria, dolor en extremidades inferiores y claudicación; así como la ingesta de medicamentos.

La exploración física debe estar encaminada a la medición de las cifras de TA, con el paciente acostado, de pie y sentado así como búsqueda de este signo en reposo y postesfuerzo isométrico (empuñadura) que manifiesten alguna causa de HAS secundaria. Como ya ha sido comentado previamente; las cifras aceptadas para el diagnóstico de HAS deben realizarse en dos visitas médicas y de ser posible con mediciones por dos observadores (médico y enfermera) y con valores $\geq 140 / 90 \mathrm{~mm} \mathrm{Hg}$. Actualmente, existen valores de cifras de tensión arterial que hacen el diagnóstico de HAS de forma ambulatoria, durante el día dichas cifras (promedio) son $\geq 135 / 85 \mathrm{~mm} \mathrm{Hg}$, durante la noche son $\geq 120 / 70 \mathrm{~mm} \mathrm{Hg}$ y durante las $24 \mathrm{~h}$ son $130 / 80 \mathrm{~mm} \mathrm{Hg}^{11}$.

Es importante mencionar que el realizar el diagnóstico de HAS únicamente no es el punto más importante, sino que es de suma importancia realizar una valoración integral para determinar daño a órgano «blanco». Dicho estudio debe incluir el electrocardiograma y ecocardiograma transtorácico para conocer el grado de hipertrofia de pared ventricular izquierda, la dilatación de cavidades auriculares y las alteraciones estructurales cardiovasculares; en algunos de estos pacientes se deberá considerar algún otro estudio para valorar la isquemia miocárdica y la anatomía de las arterias coronarias.

Se considera conveniente estudiar el daño por aterosclerosis en vasos arteriales sistémicos por lo que se debe realizar ultrasonido carotídeo para medir el grosor de la íntima de estas arterias. En este mismo contexto también se debe valorar la elasticidad arterial por medio de un USG Doppler para cuantificación de la velocidad de pulso de onda en grandes arterias y el índice brazo/tobillo. A nivel renal es importante determinar el cálculo de la función renal con la cistatina $C$ y el potasio sérico, la depuración de creatinina y la microalbuminuria en orina de $24 \mathrm{~h}$, que también complementan la evaluación de la magnitud del daño renal; la albuminuria de 30 a $300 \mathrm{mg}$ en orina de $24 \mathrm{~h}$ se denomina microalbuminuria además de la connotación de la glomerulopatía, también es un buen predictor de lesión celular endotelial sistémica, especialmente las cardiocerebrales.

Se recomienda la valoración vascular de la retina por medio de fondo de ojo; para investigar el grado de retinopatía, clasificación de Wagener, Keith y Baker y el cual se encuentra los grado 1, 2, 3 y 4 están muy relacionado con incremento de daño vascular cardiocerebrorrenal; la correlación principalmente se hace entre la fundoscopía y la enfermedad renocerebral vascular.
En el análisis del sistema nervioso central en la actualidad la información que da la tomografía computada de cráneo está siendo desplazada por la resonancia magnética cerebral, la cual determina en pacientes asintomáticos la presencia de infartos pequeños y profundos infartos, lacunares, con diámetros mayores a $3 \mathrm{~mm}$; que se encuentran con una incidencia que varía de 10 hasta en un $30 \%$ de pacientes asintomáticos $^{11}$.

\section{Tratamiento y metas}

En la actualidad el tratamiento se debe individualizar de acuerdo a comorbilidades, grado de HAS y edad; así como las metas recomendadas en las guías más recientes. En 2013 la European Society of Cardiology publicó las guías clínicas para el manejo de pacientes con HAS. Dicho manejo se individualiza de acuerdo al grado de HAS y número de factores de riesgo cardiovascular. Se debe iniciar el tratamiento con cambios en el estilo de vida, hábitos alimenticios, los cuales consisten en restricción de la ingesta de sal (cloruro de sodio (sazonador) o benzoato de sodio (conservador) (5-6 g/día), disminuir el consumo de alcohol (20-30 g/día en hombres y 10-20 g/día en mujeres), consumir una dieta de calidad «mediterránea» para conseguir una reducción de peso corporal y de la circunferencia de cintura (IMC $25 \mathrm{Kg} / \mathrm{m}^{2}, 102 \mathrm{~cm}$ en hombres y $88 \mathrm{~cm}$ en mujeres) realizar con regularidad ejercicio aeróbico cardiovascular de intensidad moderada (40 minutos al día de al menos 5 días por la semana) y abandonar el consumo de tabaco ${ }^{11,14}$.

Posteriormente y de acuerdo al grado de HAS, así como número de factores de riesgo se debe iniciar tratamiento farmacológico para obtener una meta de $<140 / 90 \mathrm{~mm} \mathrm{Hg}$, para lo cual se encuentran medicamentos como diuréticos (tiazidas/clortalidona), beta-bloqueadores cardioselectivos (prololes), calcioantagonistas (dipinos), inhibidor de la enzima convertidora de angiotensina (pril) o (IECA) y bloqueador del receptor de angiotensina (ARA); todos son adecuados para iniciar y continuar tratamiento antihipertensivo, ya sea en monoterapia o en combinación; únicamente no se recomienda combinación de dos IECA o priles ${ }^{11,14}$.

También en diciembre de 2013 el Eighth Joint National Commitee publicó la guía para manejo de la HAS; donde se realizaron varias recomendaciones dentro de las cuales se indica iniciar manejo farmacológico con cifras de tensión arterial $\geq 150 / 90 \mathrm{~mm} \mathrm{Hg}$ en pacientes mayores de 60 años y la meta de tratamiento será mantener las cifras $<150 / 90 \mathrm{~mm}$ $\mathrm{Hg}$, sin embargo si en dicha población con tratamiento farmacológico se registran cifras de presión sistólica menores de $140 \mathrm{~mm} \mathrm{Hg}$ y no se encuentran efectos adversos, el tratamiento no tiene que ser reajustado ${ }^{2}$.

En pacientes con edad $<60$ años (18 a 59 años) el inicio de tratamiento farmacológico debe iniciar con cifras $\geq 140 / 90 \mathrm{~mm} \mathrm{Hg}$ y las metas terapéuticas son mantener cifras $<140 / 90 \mathrm{~mm} \mathrm{Hg}$. En los pacientes $\geq 18$ años con diagnóstico de enfermedad renal crónica y diabetes mellitus, el inicio de manejo y la obtención de metas de control son las mismas que en la población general ${ }^{2,15}$. En el control terapéutico farmacológico inicial en la población general se deberá incluir diurético tipo tiazídico más un dipino $o$ un IECA o un sartan. Sin embargo, en la población de raza negra se sugiere iniciar manejo con diurético tipo tiazida o dipino. 
En los pacientes con HAS e IRC se sugiere iniciar manejo con IECA o $\operatorname{sartan}^{15}$.

Además se enfatiza que el principal objetivo del tratamiento es mantener la meta según la población. Si dicha meta no se logra dentro del primer mes de tratamiento, debemos ajustar el tratamiento farmacológico ya sea incrementando la dosis o agregar un segundo antihipertensivo; posteriormente en caso de un inadecuado control se debe valorar agregar un tercer antihipertensivo. Dentro de esta combinación de antihipertensivos; igualmente se sugiere no combinar sartan con IECA ${ }^{2}$.

Es importante mencionar que en el caso clínico el paciente ya presenta complicaciones crónicas (CHAS, FA y EVC); dentro del tratamiento de las mismas debería incluir anticoagulación oral. La prevención del riesgo tromboembólico es quizá la intervención médica que más repercusión positiva tenga sobre el prónostico a largo plazo del paciente con FA. Desde el inicio de la presentación se debe evaluar el riesgo individual para desarrollar tromboembolismo. Actualmente la manera correcta sobre valorar dicho riesgo incluye la clasificación de $\mathrm{CHADS}_{2}$ y $\mathrm{CHA}_{2} \mathrm{DS}_{2}$-VASC; siendo esta última la más completa y eficaz para determinar el riesgo ${ }^{16,17}$.

La anticoagulación oral con antagonistas de vitamina $\mathrm{K}$, también llamados cumarínicos; se asocia con una disminución en el riesgo tromboembólico entre 50 a $80 \%$ de riesgo relativo de EVC en los pacientes con $\mathrm{FA}^{18}$. En el momento actual se cuenta con nuevos anticoagulantes orales: los antagonistas del factor lla de la coagulación y los antagonistas del factor Xa; cuyas indicaciones terapéuticas cada vez toman más valor.

\section{Conclusiones}

Es de suma importancia una adecuada historia clínica y exploración física para realizar diagnóstico oportuno ya que en México en el año 2008 se documentó que existe una prevalencia media del $31 \%$ con una tendencia creciente; por lo que es un padecimiento que requiere especial atención ${ }^{14}$.

El cambio en el estilo de vida y alimentación, son sin duda piedra angular en el tratamiento de los pacientes hipertensos. Una terapéutica farmacológica óptima consiste en la elección de los fármacos que potencialmente ofrezcan el mayor beneficio al enfermo de acuerdo a sus características clínicas y comorbilidades.

El diagnóstico oportuno y adecuado cumplimiento de metas terapéuticas se asocia a disminución en el riesgo de complicaciones crónicas, mejor calidad de vida y disminución en costo económico.

\section{Financiación}

No se recibió patrocinio de ningún tipo para llevar a cabo este artículo.

\section{Conflicto de intereses}

Los autores declaran no tener ningún conflicto de intereses.

\section{Bibliografía}

1. Egan BM, Zhao Y, Axon RN. US trends in prevalence, awareness, treatment, and control of hypertension, 1988-2008. JAMA. 2010;303:2043-50.

2. James P, Oparil S, Carter BL, et al. 2014 Evidence-based guideline for the management of high blood pressure in adults: Report from the panel members appointed to the eighth Joint National Committee (JNC 8). JAMA. 2013;1097:1-14.

3. Chobanian AV, Bakris GL, Black HR, et al. The seventh report of the Joint National Committee on prevention, detection, evaluation, and treatment of high blood pressure: the JNC 7 report. JAMA. 2003;289:2560-72.

4. Burgaz A, Orsini N, Larsson SC, et al. Blood 25-hydroxyvitamin D concentration and hypertension: a meta-analysis. J. Hypertens. 2011;29:636-45.

5. De Simone G, Devereux RB, Chinali M, et al. Risk factors for arterial hypertension in adults with initial optimal blood pressure: the Strong Heart Study. Hypertension. 2006;47:162-7.

6. Velázquez-Monroy $\mathrm{O}$, Rosas M, Lara A, et al. Prevalence and interrelations of no communicable chronic disease and cardiovascular risk factors in Mexico. Arch Cardiol Mex. 2003:62-77.

7. Kurella Tamura M, Xie D, Yaffe K, Cohen DL, et al. Vascular risk factors and cognitive impairment in chronic kidney disease: the Chronic Renal Insufficiency Cohort (CRIC) study. Clin. J. Am. Soc. Nephrol. 2011;6:248-56.

8. Inzitari D, Eliasziw M, Gates P, et al. The causes and risk of stroke in patients with asymptomatic internal-carotid-artery stenosis. North American Symptomatic Carotid Endarterectomy Trial Collaborators. N. Engl. J. Med. 2000;342:1693-700.

9. Lorell BH, Carabello BA. Left ventricular hypertrophy: pathogenesis, detection, and prognosis. Circulation. 2000;102:470-9.

10. McKee PA, Castelli WP, McNamara PM, et al. The natural history of congestive heart failure: the Framingham study. N. Engl. J. Med. 1971;285:1441-6.

11. Mancia G, De Backer G, Dominiczak A, et al. ESH/ESC 2007 Guidelines for the management of arterial hypertension. Rev. Esp. Cardiol. 2007:60, 968.e1-e94.

12. Pickering G, Hypertension GP. Definitions, natural histories and consequences. Am J Med. 1972;52:570-83.

13. Davis JT, Rao F, Naqshbandi D, et al. Autonomic and hemodynamic origins of pre-hypertension: Central role of heredity. J. Am. Coll. Cardiol. 2012;59:2206-16.

14. Rosas M, Pastelín $G$, Vargas-Alarcón $G$, et al. Guías clínicas para la detección, prevención, diagnóstico y tratamiento de la hipertensión arterial sistémica en México 2008. Comité Institucional de Expertos en Hipertensión Arterial Sistémica. Arch Cardiol Mex 2008: 78:supl.2; abril-junio. S2-9 a S2108.

15. Pérez-Fernández GA, Grau-Abalo R. Conversión del adolescente pre-hipertenso al adulto hipertenso. Arch Cardiol Mex. 2012;82:112-9.

16. Camm AJ, Kirchhof P, Lip GY.H, et al. Guiás de práctica clińica para el manejo de la fibrilacioń auricular. Rev Esp Cardiol 2010;63(12):1483.e1-e83.

17. Fuster V, Rydén LE, Cannom DS, et al. 2011 ACCF/AHA/HRS focused updates incorporated into the ACC/AHA/ESC 2006 guidelines for the management of patients with atrial fibrillation: a report of the American College of Cardiology Foundation/American Heart Association Task Force on Practice Guidelines. Circulation. 2011;123:e269-367.

18. Olesen JB, Lip GY, Hansen ML, et al. Validation of risk stratification schemes for predicting stroke and thromboembolism in patients with atrial fibrillation: nationwide cohort study. BMJ. 2011;342:d124. 